\title{
Leichtbau immer aktueller
}

Die K 2016 in Düsseldorf ist im Oktober erfolgreich abgeschlossen worden, und gut einen Monat später startet am 29. November die Composites Europe am gleichen Standort. Allein an den vielen Veranstaltungen in diesem Jahr zeigt sich, wie der Leichtbau ständig an Bedeutung gewinnt.

Während sich die „K“ auch mit „Vorgeschalteten“ Fragen befasste, zum Beispiel wie bei den hohen Energiekosten in Deutschland Polymere nachhaltig gewonnen und eine funktionierende Versorgungskette bei schwachem Euro und der starken Nachfrage in den USA und Asien - Stichwort Rohstoffbeschaffung - erhalten werden können, wird sich die „Composites" mit rund 350 Ausstellern über das Material Kunststoff hinaus wieder mit dem gesamten Trend zu modernen Multimaterialsystemen hin beschäftigen. In diesem Jahr läuft parallel dazu die Aluminium mit rund 950 Ausstellern. Veranstalter Reed Exhibitions spricht bei dem gebotenen Ausstellungs- und Vortragsforum von einer materialübergreifenden Schnittstelle von Metall- und Faserverbundtechnologien in Strukturbauteilen.

Die Frage, wie bei komplexen faserverstärkten Bauteilen eine qualitätsgesicherte Serienfertigung erreicht werden kann, beschäftigt die Branche permanent und gilt nach wie vor als limitierender Faktor. Erste Lösungen sollen nun in Düsseldorf präsentiert werden. „Künftig wird es darum gehen, mit modernsten Produktions- und Automatisierungslösungen die Serienfertigung sicherzustellen", sagt auch der Event Director der Composites Europe,
Olaf Freier. So wird beispielsweise die additive Fertigung immer öfter genannt, auch wenn ein Markt dafür nur zögerlich entsteht - unter anderem wegen nicht sichergestellten Qualitätsstandards und verbesserungsfähiger Serientauglichkeit. Zügiger dagegen scheint das Thema Industrie $4.0 \mathrm{zu}$ greifen, bei dem eine vernetzte Produktion für die schnelle Herstellung und damit für bessere Konkurrenzfähigkeit in einer beschleunigten Welt sorgen soll. Reed erwartet in Düsseldorf rund 30.000 Fachleute aus Industrie, Forschung und Entwicklung.

Im Leichtbau interagieren zahlreiche Fachdisziplinen. Wie gewohnt, finden Sie auch in diesem Heft eine breite Palette an Themen, die die Branche beschäftigen. Doch eins noch in eigener Sache: Von dieser Ausgabe der lightweight design wird es auch eine englische Version geben. Diese erscheint zusätzlich zu diesem Heft in einer Teilauflage von 3000 Exemplaren in Print und vom kommenden Jahr an rein elektronisch. Wir möchten damit unseren Leserkreis vergrößern und sind uns sicher, das internationale Informationsbedürfnis damit noch besser bedienen zu können. Die deutsche Ausgabe bekommen Sie natürlich weiterhin gedruckt.

Wegen des ungewöhnlichen Termins der Composites Europe 2016 haben wir den Erscheinungstermin der letzten Ausgabe in diesem Jahr auf den 18. November vorverlegt. An dieser Stelle wünsche ich Ihnen schon heute einen erholsamen Jahresausklang.

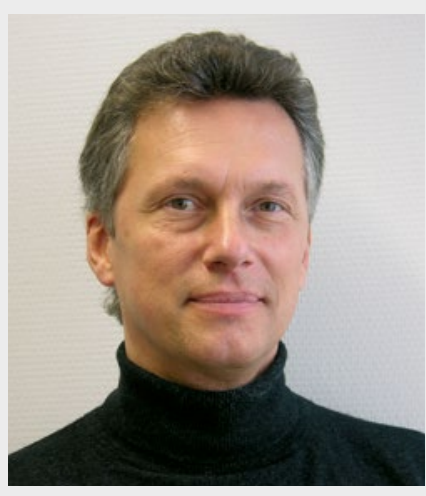

Dipl.-Ing. Ulrich Knorra Redakteur, ulrich.knorra@springer.com 